\title{
La educación científica de alumnos con discapacidad visual: un análisis en el contexto mexicano
}

\section{The scientific education of students with visual impairments: An analysis in the Mexican context}

\author{
CRISTINA G. REYNAGA-PEÑA* \\ JUAN MANUEl FERNÁNDEZ-CÁRDENAS ${ }^{* *}$
}

Este trabajo tiene como propósito estudiar los procesos de la educación científica de alumnos con discapacidad visual en educación básica a fin de identificar los factores que se requieren para lograr una educación inclusiva de las ciencias. Aunque la educación científica y tecnológica se considera indispensable para la toma de decisiones informadas en la vida cotidiana, no existe documentación en el contexto mexicano que permita dar cuenta de cómo sucede esta para los alumnos con alguna discapacidad. El análisis, realizado desde la tradición cualitativa, busca también dar voz a los actores que intervienen en el proceso: estudiantes, docentes, directivos y formadores de docentes. Los datos muestran que se trata de un asunto multifactorial y complejo con áreas de oportunidad en la articulación del currículo, en la preparación de los docentes y sus condiciones de trabajo, e incluso en la participación de la familia en el proceso educativo.

This work aims to understand the processes that take place during scientific education of students with visual impairments at the middle school level, with the objective of identifying what is needed for an inclusive science education to be achieved. Although scientific and technological education is considered essential for making informed decisions in everyday life, there is no documentation in the Mexican context that reports how science education takes place for students with disabilities. This analysis, carried out within the qualitative tradition, seeks to give voice to the actors who participate in the process: Students, teachers, administrators and teachers of teacher development programs. Data shows that inclusive science education for students with visual impairment is a multifactorial and complex process that holds challenges in the curriculum implementation, teacher preparation, teacher's work conditions, and even participation of the family.
Palabras clave:

educación científica, educación inclusiva, discapacidad visual, currículo

\section{Keywords:}

scientific education, inclusive education, visual impairments, curriculum

Recibido: 15 de febrero de 2019. | Aceptado para su publicación: 1 de agosto de 2019. Recuperado de: https://sinectica.iteso.mx/index.php/SINECTICA/article/view/968 doi: 10.31391/S2007-7033(2019)0053-007

Sección: Investigaciones temáticas

\footnotetext{
* Doctora en Genética por la Universidad de California, Riverside (EUA). Profesora-investigadora de la Escuela de Humanidades y Educación en el Tecnológico de Monterrey, Campus Monterrey. Pertenece al Sistema Nacional de Investigadores del Conacyt, nivel 1. Línea de investigación: educación científica de poblaciones en situación de marginalidad y formación docente, abordados desde el enfoque de la educación inclusiva de las ciencias. Correo electrónico: cristina.reynaga@tec.mx/orcid.org/0000-0003-3039-551X

** Doctor en Educación y Lingüística por la Open University, Reino Unido. Profesor-investigador de la Escuela de Humanidades y Educación y director de la maestría en Tecnología Educativa del Tecnológico de Monterrey. Es miembro del Sistema Nacional de Investigadores del Conacyt, nivel I. Líneas de investigación: conformación de comunidades de práctica relacionadas con las actividades de educación en STEM, evaluación de la calidad educativa y análisis del habla en interacción en escenarios educativos mediados por el uso de tecnología digital, en particular en temas de desarrollo moral, cultura de la legalidad, lectoescritura y educación en ciencias. Correo electrónico: j.m.fernandez@ tec.mx/orcid.org/0000-0003-2044-1658
} 


\section{INTRODUCCIón}

\section{La educación inclusiva}

L a Convención de los Derechos de Personas con Discapacidad (United Nations, 2006) reconoce el derecho de los niños con alguna discapacidad de ser incluidos en los sistemas de educación general. En específico, el artículo 24 de este documento acentúa la necesidad de que los gobiernos puedan proveer un acceso igualitario a un sistema de educación inclusiva en todos los niveles educativos, a la vez de proveer el apoyo individual que los alumnos requieran. De acuerdo con el reporte de la Organización Mundial de la Salud sobre la discapacidad, en el cual se aborda el tema de la educación para las personas con discapacidad de una manera detallada, muchos niños y adultos con discapacidad han sido históricamente excluidos de las oportunidades de educación regulares (World Health Organization [WHO], 2011, p. 205].

Este reporte hace hincapié en que debe ser prioridad en todos los países el asegurar que estos niños reciban una educación de calidad en ambientes inclusivos con la intención de lograr el objetivo del desarrollo del milenio de una educación para todos, como se establece en el marco de acción de la Convención de Dakar de la Organización de las Naciones Unidas para la Educación, la Ciencia y la Cultura (Unesco, 2000, 2009).

La educación inclusiva, en un sentido estricto, significa que todos los niños con discapacidad, o sin ella, deben educarse en aulas regulares, con compañeros de su misma edad. Esta aproximación acentúa la necesidad de que todo el sistema educativo debe ajustarse para que esto suceda, ya que implica que se identifiquen y eliminen las posibles barreras, a la vez de proveer de adaptaciones razonables para las necesidades especiales que pueda tener cada alumno; esto, con la finalidad de permitir que los alumnos con alguna discapacidad participen y avancen dentro de los mismos espacios educativos que sus coetáneos. Con base en estos lineamientos, el reporte reconoce que en países en desarrollo el movimiento hacia la educación inclusiva va comenzando, ya que, en la práctica, es difícil asegurar una inclusión plena de todos los alumnos (WHO, 2011).

A pesar de los avances en este tema en décadas recientes, a nivel mundial, los niños y jóvenes con discapacidad tienen nivel de escolaridad más bajo, y menos posibilidades de lograr niveles superiores de educación. En esto, México no es la excepción, ya que el reporte más reciente del Instituto Nacional para la Evaluación de la Educación (INEE, 2015, p.121) muestra que, entre las poblaciones vulnerables, las personas con alguna discapacidad poseen el promedio de escolaridad más bajo. De acuerdo con el Censo Nacional de Población y Vivienda en México, el nivel promedio de escolaridad reportado para las personas con discapacidad visual mayores de quince años es de 3.7 años, promedio mucho menor que el de la población en general, estimado en 9.4 años (INEGI, 2010).

Es inobjetable que la educación básica para cualquier persona es importante por sí misma, pero, desde una perspectiva amplia, la falta de esta tiene un impacto significativo en la pobreza de las personas cuando son adultos, ya que disminuye sus posibilidades de lograr mejores empleos. Esto se acentúa aún más en el caso de las personas con alguna discapacidad. 


\section{IMPORTANCIA DE LA EDUCACIÓN CIENTÍFICA Y TECNOLÓGICA}

Una meta de la educación científica y tecnológica actual es el ingreso de las personas a la sociedad del conocimiento, como motor de la prosperidad económica y para lograr un mejor nivel de vida. Esta educación también es indispensable para la toma de decisiones informadas en la vida cotidiana. Por ello, en los países anglosajones, la educación en ciencia, tecnología, ingeniería y matemáticas (STEM, por sus siglas en inglés) se ha perfilado como un eje de máxima relevancia hacia el cual esos países han dirigido recursos y acciones importantes desde sus políticas públicas en los últimos años.

Comparativamente, la educación en nuestro país ha priorizado la educación en áreas concernientes al lenguaje (español) y a las matemáticas. En su reporte sobre la educación en ciencias a nivel de educación básica, Flores-Camacho y colaboradores (2012) encontraron que, a lo largo de la historia de la educación básica en México, las ciencias han ocupado un papel secundario, y que las ciencias naturales son consideradas un asunto complementario, no esencial y no útil para la vida cotidiana, por lo que se les dedica menos tiempo y recursos.

Si esto ocurre en general, el caso de las personas con discapacidad visual es más preocupante, ya que la educación científica y tecnológica para ellos es casi inalcanzable. Algunos de los factores que influyen es que, tradicionalmente, la enseñanza de las ciencias experimentales se apoya en recursos visuales que son inaccesibles para el alumno ciego y poco ilustrativos para el débil visual. Por su parte, las actividades experimentales bien diseñadas son un recurso didáctico indispensable para la educación científica, pero las propuestas específicas para alumnos con discapacidad visual son escasas (López et al., 2017; Reynaga et al., 2014); la mayoría han sido generadas en otros países (por ejemplo, Linn, 1972; Malone \& De Lucchi, 1979; American Chemical Society, 2001; Stefanich, 2001) y en español existe solo un libro (Soler-Martí, 1999).

Existen reportes que indican que los profesores que asumen la educación inclusiva consideran que las clases de ciencia se prestan, especialmente, para la inclusión de estudiantes con discapacidades (Atwood \& Oldham, 1985; McGinnis, 2013), por lo que el potencial de una enseñanza inclusiva de la ciencia es un tema por considerar. Además, en virtud de que el más reciente Modelo Educativo para la Educación Obligatoria (Secretaría de Educación Pública, 2017) prevé la inclusión como uno de sus ejes principales, cobra especial relevancia documentar lo que ocurre en la actualidad en la educación inclusiva de las ciencias en educación básica.

En este trabajo, abordamos el caso de las personas con discapacidad visual, la segunda forma de discapacidad más numerosa en México, como un ejemplo paradigmático que nos permite indagar cómo sucede la educación inclusiva de las ciencias en escuelas regulares del sistema de educación pública a nivel de educación básica. De nuestro conocimiento, no existen investigaciones en el contexto mexicano que den cuenta del estado actual de la educación en ciencia y tecnología para este grupo vulnerable.

Así, el estudio aquí presentado tiene como objetivo responder a las siguientes preguntas de investigación: ¿qué ocurre en el contexto escolar respecto a la educación inclusiva en ciencia y tecnología y, en particular cuando se trata de la inclusión de personas con discapacidad visual en actividades de ciencia? ¿Cómo se compara la propuesta curricular y los marcos de las políticas públicas vigentes con la realidad de las escuelas? En virtud de que en las propuestas educativas más recientes se 
reconoce el papel del docente, surge también la pregunta ¿de qué manera esto se relaciona con el papel del docente, incluyendo la formación o actualización docente?

\section{MARCO TEÓRICO Y CONCEPTUAL}

\section{Cognición distribuida como un enfoque para la educación con una perspectiva integradora}

En este artículo, decidimos asumir una perspectiva sociocultural, en particular desde la teoría de la cognición distribuida, para interpretar los datos que aquí presentamos. Esto, dado que con esta perspectiva se centra la atención en los atributos y las restricciones de los sistemas de actividad humana, el rol de las herramientas y de las trayectorias de los participantes para construir sus identidades. La teoría de la cognición distribuida (Wilson, 2002) posee como principal argumento que, en vez de tener un agente central que piensa sobre cómo actuar en sus propias circunstancias, existe un sistema de actividad situado que articula los esfuerzos de los participantes que se encauzan hacia el logro de metas compartidas en dicho sistema.

Ejemplos prototípicos de cognición distribuida incluyen el estudio de la navegación de grandes embarcaciones (Hutchins, 1995), laboratorios de geoquímica (Goodwin, 1997), hospitales (Engeström, 2005) y, por supuesto, entornos educativos formales y no formales (Cole, 1998; Gutiérrez \& Vossoughi, 2010). En todos estos casos, la teoría de la cognición distribuida destaca las interpelaciones entre cognición, cultura y herramientas, y reconoce la importancia tanto de un guía (docente, contramaestre, jefe de laboratorio) como de los artefactos cognitivos que rodean a la persona involucrada en el aprendizaje. Así, las escuelas y los salones con alumnos incluidos constituyen un ejemplo de actividad que es susceptible a este tipo de análisis.

La conceptualización del aprendizaje de la ciencia desde la perspectiva de la cognición distribuida también invoca la reconsideración del papel del medio ambiente en general y de los artefactos, en particular en la realización de actividades cognitivas. Debemos subrayar que el lenguaje en sí mismo es una herramienta social a través de la cual los humanos median su relación con el mundo, cuyo significado solo se puede comprender al referirse a la actividad en la que están involucrados.

Al concebir el lenguaje como un artefacto que los participantes emplean para cumplir sus tareas, el conocimiento se construye con la participación en un conjunto de fenómenos, objetos y procesos heterogéneos que forman parte de la actividad cognitiva de los estudiantes. Al expandir su enfoque de análisis más allá de los límites de un solo individuo, la cognición distribuida tiene el potencial de permitirnos identificar cómo interactúan los estudiantes entre sí y con artefactos para elaborar sus tareas, y analizar cómo se construyen los significados compartidos en estas interacciones.

\section{Metodología}

En este trabajo utilizamos métodos de análisis cualitativo (Maxwell, 2013). Los datos fueron obtenidos por medio de análisis de dos documentos de políticas públicas en México, entrevistas semiestructuradas, y observación de actividades en dos escuelas públicas del estado de Nuevo León, a partir de las cuales se generaron notas de campo. 


\section{Participantes y contexto}

Para el estudio, realizamos entrevistas semiestructuradas individuales a:

-Dos jóvenes con discapacidad visual. Uno de ellos cursaba el primer año de educación secundaria en una escuela pública en una población cercana a Monterrey al momento de la entrevista (EE2). El segundo es un joven recién graduado de la universidad (EE1). Ambos fueron entrevistados para dar cuenta de su trayectoria escolar en el nivel educación básica.

-Cinco profesores que laboran en distintos puestos en el sistema educativo, cuyas características se describen en la tabla.

Tabla. Descripción de los docentes participantes en las entrevistas

\begin{tabular}{|c|c|c|}
\hline Código & Descripción & Tipo de escuela o sistema donde labora \\
\hline ED1 & $\begin{array}{c}\text { Docente con más de veinticinco años de servicio } \\
\text { en el sistema educativo (educación básica, educa- } \\
\text { ción especial y educación universitaria). Labora } \\
\text { como formador de docentes y responsable de } \\
\text { programas en el Sistema de Educación Especial }\end{array}$ & $\begin{array}{c}\text { Escuela Normal de Especialización y } \\
\text { Sistema de Educación Especial de la Secre- } \\
\text { taría de Educación Pública en el Estado }\end{array}$ \\
\hline ED2 & $\begin{array}{c}\text { Docente con más de treinta años de servicio en } \\
\text { el sistema educativo; colabora en el Sistema de } \\
\text { Educación Especial en Nuevo León }\end{array}$ & $\begin{array}{c}\text { Sistema de Educación Especial de la } \\
\text { Secretaría de Educación Pública }\end{array}$ \\
\hline ED3 & $\begin{array}{c}\text { Docente de apoyo, perteneciente al Sistema de } \\
\text { Educación Especial, con tres años de servicio }\end{array}$ & $\begin{array}{c}\text { Escuela secundaria pública ubicada en una } \\
\text { población cercana a la ciudad de Monte- } \\
\text { rrey, Nuevo León }\end{array}$ \\
\hline ED4 & $\begin{array}{c}\text { Docente que imparte la asignatura de matemáti- } \\
\text { cas a nivel secundaria, con seis años de servicio }\end{array}$ & $\begin{array}{c}\text { Escuela secundaria pública ubicada en una } \\
\text { población cercana a la ciudad de } \\
\text { Monterrey, Nuevo León }\end{array}$ \\
\hline ED5 & $\begin{array}{c}\text { Docente que imparte la asignatura de Biología en } \\
\text { secundaria, con más de veinte años de } \\
\text { servicio en el sistema educativo }\end{array}$ & $\begin{array}{c}\text { Escuela secundaria pública ubicada dentro } \\
\text { del área metropolitana de } \\
\text { Monterrey, Nuevo León }\end{array}$ \\
\hline ED6 & $\begin{array}{c}\text { Docente de apoyo, perteneciente al Sistema de } \\
\text { Educación Especial, con quince años de servicio }\end{array}$ & $\begin{array}{c}\text { Escuela secundaria pública ubicada dentro } \\
\text { del área metropolitana de } \\
\text { Monterrey, Nuevo León }\end{array}$ \\
\hline
\end{tabular}

Las entrevistas fueron grabadas con el permiso de los participantes, transcritas y examinadas por medio de un programa de análisis cualitativo. El análisis de la información se fundamentó en la teoría fundamentada (Corbin \& Strauss, 1990), en la cual las hipótesis se generan a partir de la información que proveen los informantes. A excepción del código que describe la articulación del currículo, el cual está basado en los documentos de políticas públicas, los códigos emergieron, sistemáticamente, a través de los datos proporcionados por los participantes y las notas de campo. Los códigos iniciales fueron agrupados en categorías que permitieron crear un sistema más robusto y transversal de la percepción de los participantes. La validación de los datos se sustentó en la triangulación de la información proveniente de las distintas fuentes.

Con la intención de ejemplificar los resultados, la codificación de las fuentes que utilizamos para este reporte es la siguiente:

-(E) para denotar datos obtenidos de entrevistas, seguidas de otra letra para identificar a cada persona entrevistada (EE, entrevista a estudiante, ED, entrevista a docente) y un tercer identificador es el número de participante.

-Empleamos el código NC para información obtenida a partir de notas de campo, seguidas de un identificador de la escuela que se visitó (G o Z). La escuela 
G corresponde a una escuela secundaria pública en un contexto urbano de nivel socioeconómico medio/bajo dentro de la zona metropolitana de Monterrey. Por su parte, la escuela Z representa a una escuela secundaria pública en una población cercana a Monterrey en un contexto socioeconómico bajo/ muy bajo. En ambos casos, el grupo observado fue uno de los de primero de secundaria, en el que se encontraban los alumnos con discapacidad visual.

El número de estudiantes en el grupo observado en la escuela Z fue de 44 por grupo, mientras que el de la G estaba compuesto por 39 alumnos. En la escuela Z, el alumno tenía discapacidad severa, mientras que en la G, la alumna tenía baja visión. Debido al enfoque de este trabajo, observamos únicamente las clases de biología y matemáticas. Cabe aclarar que las notas de campo incluyen también observaciones de una visita a un laboratorio de ciencias en la escuela $\mathrm{Z}$ y de conversaciones informales con los docentes de educación básica y especial, con los responsables de laboratorios y con los directivos de la escuela.

\section{RESULTADOS}

Las categorías generadas a partir del análisis de los datos permitieron la identificación de los diversos factores que intervienen en la educación inclusiva de las áreas de ciencia y matemáticas, y que se comparten con la educación inclusiva en general. Estas categorías son descritas a continuación:

\section{Articulación del currículo}

En el acuerdo 592, mediante el cual se articula la Reforma Integral de la Educación Básica en 2011 (Secretaría de Educación Pública, 2011), la inclusión es uno de los objetivos que se destaca en el discurso. Primero, en la descripción de sus características queda de manifiesto que (el acuerdo 592) "es una política pública que impulsa la formación integral de todos los alumnos de preescolar, primaria y secundaria" y que requiere "favorecer la educación inclusiva" (Secretaría de Educación Pública, 2011, p. 6). Así, la inclusión se presenta como parte de uno de los principios pedagógicos inscritos: "1.8. Favorecer la inclusión para atender la diversidad".

Sin embargo, los datos encontrados a partir del análisis de entrevistas y la observación en escuelas indican que eso no siempre sucede. Uno de los entrevistados, con amplia trayectoria como docente de educación especial y experiencia en distintos puestos dentro del sistema educativo, comentó acerca del principio 1.8: "Pero eso no se hace realidad casi nunca; se ven todos los principios pedagógicos, pero ese se brinca" (ED1).

Por su parte, en el documento del más reciente Modelo Educativo para la Educación Obligatoria (Secretaría de Educación Pública, 2017) se plantea "la inclusión y equidad como principios básicos transversales, lo cual exige que la formación inicial adopte una perspectiva equitativa, inclusiva e intercultural" (p. 143). De hecho, se menciona que "la plena inclusión de estudiantes con discapacidad que plantea el Modelo supone que todos los maestros deben contar con preparación en la atención de niñas, niños y jóvenes en esta condición" (pp.143-144). 
A través de las entrevistas, y en opinión de los mismos informantes, se sugiere que, para que esto ocurra, el sistema actual debe cambiar y que ello puede tomar tiempo:

Si hablamos de educación inclusiva "tenemos que cambiar el sistema, tenemos que modificar todo para que este niño y todos funcionen", o sea, una escuela para todos (ED1).

Con las condiciones actuales sobre el acceso a la educación está generándose una actitud y unas políticas de trabajo que empujan hacia una mayor flexibilidad, hacia un mayor respeto a la diversidad, a tratar de hacer realmente una escuela para todos. Pero eso sigue siendo un ideal, como es nuestro artículo tercero. Sigue siendo, en parte, un deseo, en parte, un camino. Pero [...] de aquí a que ocurra, pues todavía falta un rato (ED2).

Como uno de los factores en esta categoría, encontramos también que existen dificultades con la comunicación y la coordinación entre docentes de educación básica y de educación especial: “... los alumnos con discapacidad, los traían, y era problema de los de educación especial el hacerlos que avanzaran” (ED1). Una profesora de apoyo del área de educación especial ejemplifica esto a su manera:

El maestro [del aula regular] me dice: "Ah no, es que yo no le explico a él, porque no sé cómo hablarle, ni cómo tratarlo, ni qué pedirle" [al alumno con discapacidad] [...], aunque yo le estoy dando un expediente, le voy a ver regularmente, le pregunto si tiene alguna duda de trabajo, o si se le está complicando alguna explicación y me dicen: "Todo bien, todo bien" [...], pero luego dicen que no saben (ED3).

Esto coincide con la información proporcionada por un docente de educación básica, quien expresa que la maestra de educación especial "es quien está a cargo de la alumna con discapacidad visual". Incluso manifiesta abiertamente no estar de acuerdo con la Secretaría de Educación en cuanto a la educación inclusiva en el aula regular, y considera que los alumnos con necesidades educativas especiales debían atenderse en escuelas especializadas para lograr su mejor desarrollo educativo (NCG).

Una de las profesoras de educación especial entrevistadas, al narrar su experiencia, afirma que los profesores del aula regular entienden mal el concepto de educación inclusiva al esperar que, por ejemplo, todos los alumnos entreguen reportes escritos por igual, en vez de permitir múltiples o diferentes formas de expresión del conocimiento, de acuerdo con sus posibilidades (NCZ, ED3). Ella comenta también que observa con frecuencia que los profesores no llevan a cabo las adecuaciones específicas que requiere el alumno, a pesar de las recomendaciones que les hace; esto se define en función de las necesidades especiales que cada alumno puede tener.

Algunos ejemplos de estas necesidades son: el tiempo que el alumno pueda requerir para ciertas actividades, su capacidad de lectura, o el lugar donde puede ubicársele de manera idónea en el aula, entre otras (NCZ, ED3). Es importante mencionar que las dos profesoras de educación especial entrevistadas describen en detalle las características y fortalezas de cada uno de los alumnos que atienden (ED3, ED6, NDZ, NDG), información que queda plasmada en los expedientes que elaboran para cada alumno. Sus relatos hacen ver que en estos expedientes queda registrada toda la información relevante relacionada con el alumno, y ejemplifican la dedicación y atención que ponen en ello (NCG, NCZ), lo que corrobora otro de los informantes (ED1).

Entre otros factores relacionados con la articulación del currículo, aparecen las condiciones de trabajo de los docentes del aula regular, como el manejo de grupos grandes, hasta de 40 alumnos o más (44 en una de las aulas visitadas), 
que les dificulta dedicar atención a la adaptación de materiales para los alumnos con discapacidad; la saturación de horas en su jornada y el no contar con tiempo suficiente para fomentar la conversación y coordinación entre el maestro del aula regular y el de apoyo (NCG, NCZ, ED3, ED4).

Algunos entrevistados refieren que, a menudo, requieren tiempo fuera del horario de trabajo para este fin (ED5, ED6) y no siempre es posible contar con este espacio (NCG, NCZ). Por parte del docente de apoyo o de educación especial, las problemáticas observadas fueron: el número de alumnos con necesidades educativas especiales que les son asignados por maestro (hasta 27, entre los cuales ocho tienen alguna discapacidad); el atender de manera simultánea a varios alumnos con discapacidades distintas; tiempo insuficiente para dar seguimiento en clase a todos los alumnos con discapacidad, así como el tiempo para llevar a cabo las evaluaciones de cada alumno, entre otras (NCZ, NCG, ED3).

Un factor adicional, relacionado con la forma en que ocurre la educación científica en el sistema de educación pública, es que el maestro de ciencias en educación básica, en general, no recurre a las actividades de experimentación como parte de sus recursos pedagógicos. Esto queda evidenciado en distintas fuentes, desde los alumnos con discapacidad visual que comentan no haber realizado experimentos de biología (EE1, EE2) hasta el registro del laboratorio de ciencias de una de las escuelas visitadas, que muestra que, a siete meses de haber iniciado el ciclo escolar, los alumnos de primer grado de secundaria no han tenido una sola práctica de biología (NCZ). Este dato es confirmado por una de las profesoras de educación especial de la misma escuela, que menciona que el grupo al cual pertenece su alumno con discapacidad visual no ha ejecutado actividades experimentales en el laboratorio durante las clases de biología y que, por lo regular, es hasta tercer año de secundaria que hacen experimentos de química en el laboratorio (ED3).

Finalmente, las observaciones en clase y datos de conversaciones informales con uno de los docentes del aula regular también revelan que los docentes no cuentan con materiales adecuados sobre temas de ciencia o matemáticas para el alumno con discapacidad visual (NCG, NCZ): "Ese tipo de material no lo tenemos y sí falta. En un momento determinado donde ellos puedan con el tacto, por ejemplo, conocer todas las partes de la célula, todos los aparatos y sistemas. 0 sea, lo básico, lo básico en ciencias naturales o biología" (ED5).

En la figura 1 ilustramos los atributos y las contradicciones de la articulación del currículo con las necesidades de la educación inclusiva de STEM. 


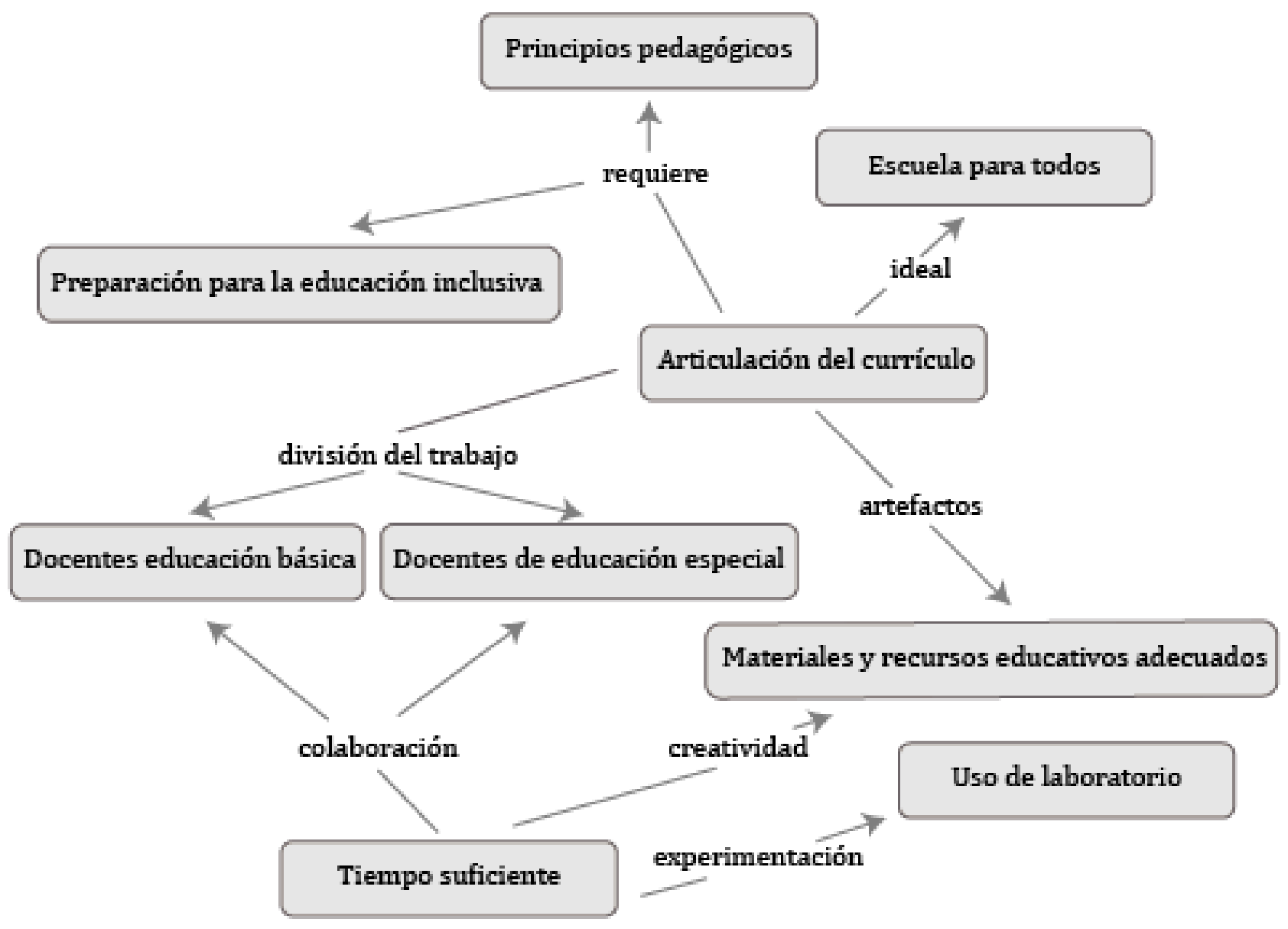

Figura 1. Articulación del currículo.

\section{La preparación de los docentes}

Varias de las fuentes de información coincidieron en que la preparación de los docentes es uno de los factores que influyen en el logro de una educación inclusiva de ciencia y tecnología, en particular cuando se trata de la participación activa de alumnos con alguna discapacidad en actividades de ciencia. La información proviene tanto desde la perspectiva del alumno como de la visión de un supervisor y, notablemente, desde la misma voz de los docentes de educación especial y educación básica.

Por un lado, se hizo referencia a la preparación de los docentes (de primaria) en cuanto al conocimiento para la enseñanza de la ciencia:

Para mí, uno de los grandes problemas es que el profe no está muy educado en ciencias. El maestro tiene una cultura general, pero no entra, no puede entrar en detalle; es decir, te explica cosas muy básicas que vienen en el libro, pero entonces eso ya genera un tropiezo, porque si un profe no le sabe a la ciencia, pues cómo la va a trabajar (ED1).

De igual manera, es evidente que el docente de educación especial tampoco se siente preparado: "Llevamos asignaturas de Didáctica del español, Didáctica de las matemáticas, pero aquí no tenemos una que se llame Didáctica de las ciencias" (ED1). Registramos también información de un profesor de matemáticas, quien dijo no estar preparado 
para enseñar a un alumno con discapacidad visual (ED4, NCZ) y de un profesor de biología que mencionó que se especializó en biología y no en educación especial (ED5, NCG).

La figura 2 presenta las relaciones entre preparación docente y los retos de la inclusión educativa.

\section{Cultura general básica en ciencia}

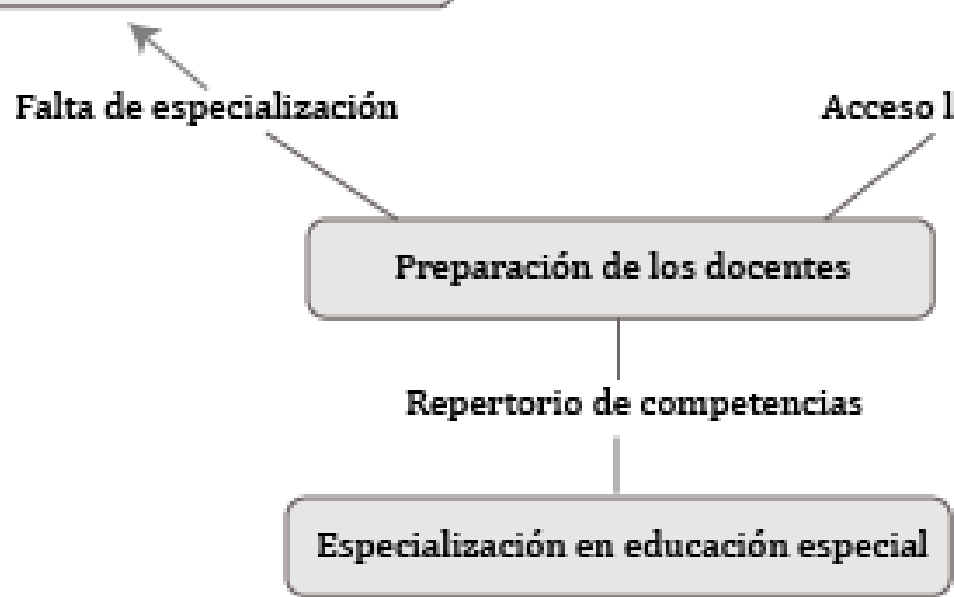

Materiales educativos básicos

Figura 2. Preparación docente.

Los retos de la educación inclusiva STEM

Una de las dificultades más comunes es la creencia generalizada de que el tener discapacidad necesariamente significa también una carencia de habilidades cognitivas o intelectuales, y que no es solo una falta de percepción sensorial que posee la persona (el sentido de la vista en el caso de las personas con problemas visuales). Así, diversas fuentes arrojan datos de que puede existir la concepción, por parte del docente, de que el alumno no puede realizar o no posee la capacidad suficiente para llevar a cabo una actividad escolar común o la construcción de conocimientos avanzados:

Sí, porque comúnmente se les trabaja mucho menos que a los de primaria, porque se cree que no entenderían (ED1).

En un CAM no se trabaja casi nada, casi cero. Se les pone a hacer algún trabajo manual o se les pone a que intenten colorear su nombre [...] cosas muy básicas (ED2).

Si eres una persona con discapacidad, este [...] al momento de que escuchar discapacidad ya piensan de que “jah no puede hacer esto!”. O está enfermo de algo (EE1).

Como posible consecuencia de esta situación, el alumno con ceguera a menudo no participa activamente en las actividades de las clases regulares o no al igual que sus compañeros (NCZ). Esto también fue manifestado por una persona adulta con discapacidad visual, en referencia a su trayectoria escolar: "[Yo] iba a la primaria, a la normal, con mis compañeros, pero solamente a perder el tiempo, yo creo, porque solamente iba a visitarlos y a estar un momento en el salón, pero realmente no hacía nada" (EE1). 
Más aún, esta percepción llega a influir en la perpetuación de la situación, como lo describe un docente entrevistado: "En realidad no se promueve una vida social, una vida de relación con el mundo, sino con un núcleo que sobreprotege, y que en lugar de ayudar ata y limita" (ED2).

Encontramos indicios de que el docente de educación básica podría mejorar su práctica con el uso de recursos y métodos pedagógicos más adecuados; por ejemplo, el docente frente a grupo, en general, utiliza el recurso de la escritura o de gráficos en el pizarrón, pero sin la incorporación de descripciones detalladas que requiere un alumno con discapacidad visual o el uso de materiales táctiles que faciliten la representación de conceptos (NCG, NCZ). Además, estudiantes con discapacidad visual reportaron que es usual que les pidan reportes escritos, y no en braille: "La maestra del grupo solamente ponía dictados" (EE1). "Los reportes los tenía que hacer en blanco y negro, y aunque yo sé escribir en blanco y negro, yo no podía escribir con letra pequeña, porque no miro lo que estoy escribiendo" (EE1).

Cabe mencionar que las barreras que puede representar el uso de recursos se disminuye cuando se trata de alumnos con baja visión, ya que, a pesar de que los libros en macrotipo (o en braille) no coinciden con el que usa el alumno regular, si es posible hacer copias que muestren la información en mayor tamaño, como lo expresa una de las docentes entrevistadas y que fue posible observar también en las clases (NCG):

Yo le doy ciencias, entonces allí no coincidió el libro que llevan los niños con el libro que lleva ella, pero se hacen las adecuaciones porque en sí son los mismos temas. Hay ocasiones en donde hay un tema que yo quiero que ella vea y que no viene en el libro, yo del libro que tengo le saco ampliación y se lo doy a ella (ED5).

En la figura 3 incluimos los desafíos de la educación STEM con una perspectiva inclusiva.

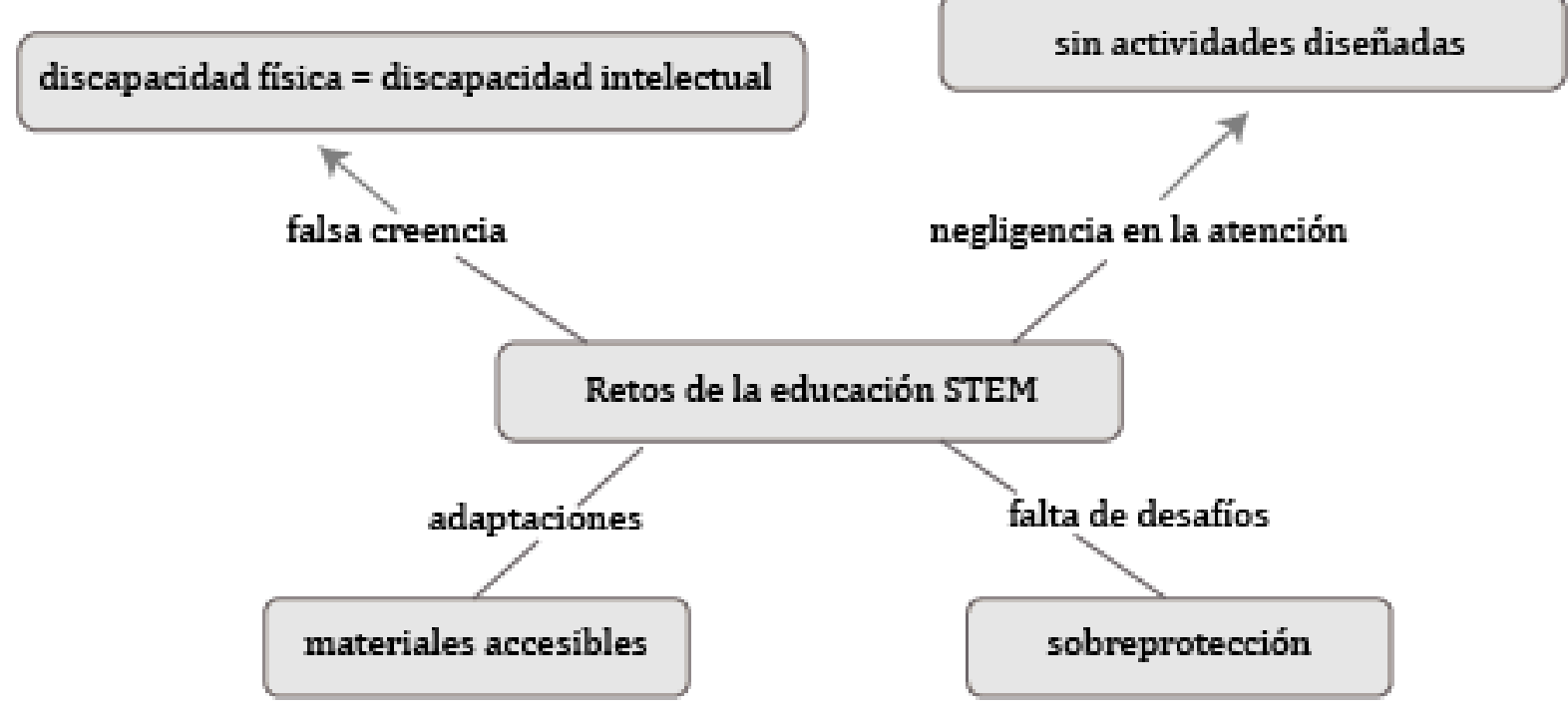

Figura 3. Retos de la educación STEM inclusiva. 


\section{El papel de la familia}

Distintas fuentes señalan que el papel de la familia se vuelve primordial en la educación de alumnos con discapacidad: desde la participación del padre o madre en la escuela: "A uno de ellos lo traía a la escuela su papá y a otro su mamá, incluso su mamá estudió algo sobre pedagogía para ayudar a su hijo" (ED6).

La familia también juega un papel esencial en el desempeño escolar, la entrega de tareas o la lectura. Debido a que con frecuencia los alumnos con discapacidad visual tienen que entregar reportes o elaborar tareas que requieren el uso de la vista, los entrevistados reportaron que sus familiares les ayudan a elaborarlos (EE1, EE2). Esta responsabilidad recae en la mamá o los hermanos, y en ocasiones en el papá: "Yo muchas veces le pedía apoyo a mi papá, a mi familia. Que me ayudara. Sobre todo, más a mi papá porque, bueno, mi mamá tiene primaria y mi papá tiene secundaria" (EE1).

En educación especial reconocen la importancia del papel de la familia en el desarrollo y desempeño escolar del alumno con necesidades educativas especiales, por lo que buscan integrarlos mediante una red de padres con reuniones periódicas (ED1). Asimismo, los profesores de educación especial que dan apoyo en las escuelas mantienen a lo largo del periodo escolar reuniones frecuentes con los padres de familia de alumnos con discapacidad a fin de conocer las características, fortalezas y retos de cada alumno con quien ellos trabajan. Esto lo expresa una de las docentes de educación especial: "Y lo que hacemos también en apoyo escolar es que los padres tengan mucho contacto con los maestros de cada materia, los acercamos mucho para que no nada más nosotras las de apoyo seamos el nexo, sino que sea un trabajo colaborativo, padres, maestros y la chica" (ED6).

Lo anterior propicia el intercambio de información de lo que sucede en la escuela y en casa para recomendar acciones y dar seguimiento activo al desarrollo escolar del alumno con necesidades educativas especiales (ED2, NCG, NCZ). La figura 4 representa las formas en las que los padres de familia se vinculan al trabajo de los docentes.

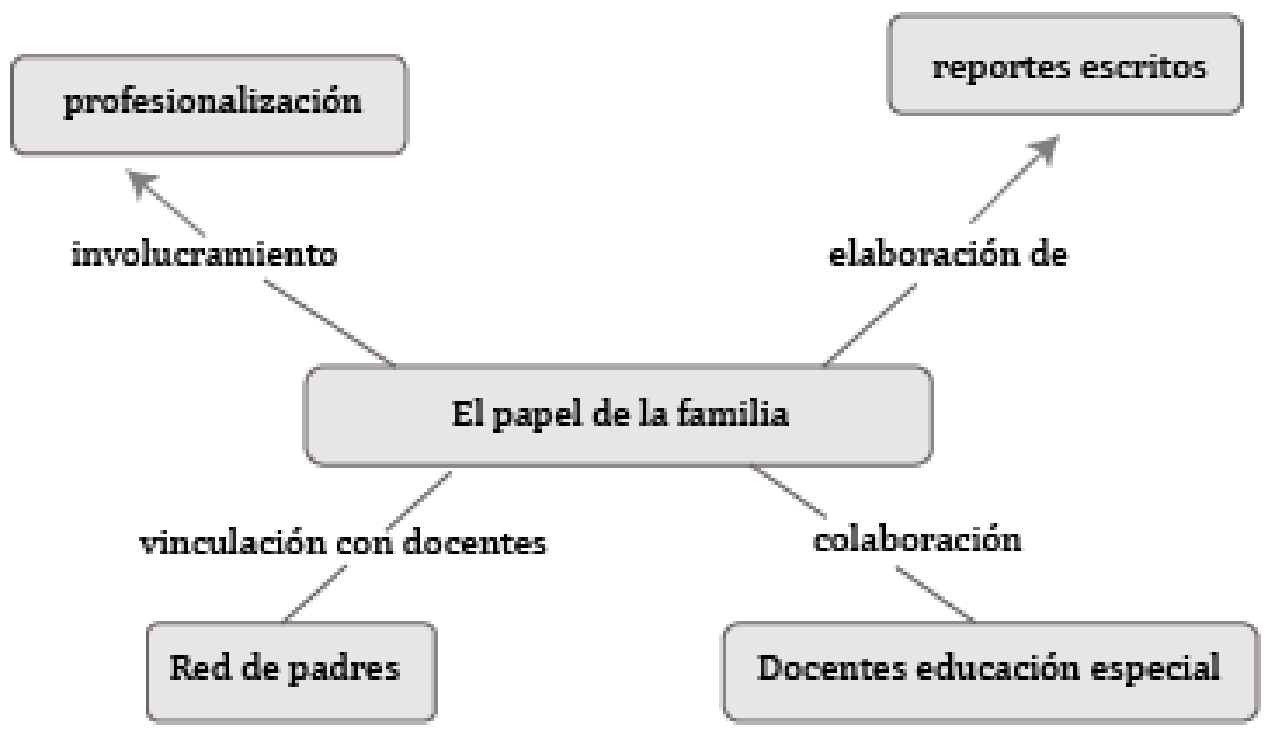

Figura 4. El papel de la familia. 


\section{CONCLUSIONES}

Por medio de este trabajo ha sido posible identificar algunos factores que, desde la voz y experiencia de los actores involucrados, intervienen en la educación inclusiva de alumnos con discapacidad visual a nivel de educación básica y, en particular, en su educación científica y tecnológica.

Uno de los factores principales que sale a la luz es la falta de preparación de los docentes del aula regular para aceptar y ejercer la educación inclusiva en general y, más aún, la educación inclusiva de las ciencias; es decir, advertimos la necesidad de actualizar los métodos pedagógicos para que estos sean más incluyentes y, de igual manera, desarrollar las competencias que les permitan identificar mejor las habilidades y capacidades de una persona con necesidades educativas especiales. Para ello, proponemos que una forma de llegar a este reconocimiento es que los docentes y las autoridades escolares se sensibilicen hacia la discapacidad de un modo vivencial que les permita, momentáneamente, ponerse en los zapatos del alumno y construir un conocimiento práctico sobre las características de su condición y cómo es posible incluir al alumno en el aula. Desde nuestra experiencia, esto es posible por medio de talleres de formación continua, que, además, tienen un impacto en la transformación de la práctica docente hacia la inclusión (Reynaga-Peña et al., 2018).

Por otro lado, suele depositarse toda o una gran parte de la responsabilidad en el docente. Sin embargo, en este análisis hemos encontrado que en realidad la educación inclusiva es un asunto multifactorial y complejo, que presenta retos desde diversas áreas. Más complejo aun cuando la inclusión se observa en relación con la educación científica y tecnológica del alumno, ya que en este apartado se potencian algunas de las dificultades y retos históricos de la educación básica; entre ellos, destacan los factores que refieren las condiciones de trabajo de los docentes, como el tamaño de los grupos, la distribución del tiempo, la falta de materiales o tiempo para prepararlos, entre otros.

Algunos de nuestros datos coinciden con los hallazgos de un reporte previo sobre los retos de la educación inclusiva en México (Romero y García, 2013). Esta situación no sucede solo en México; de acuerdo con el reporte mundial sobre discapacidad en el mundo, la educación inclusiva para las personas con discapacidad enfrenta retos o barreras que van desde problemas en el sistema (división de responsabilidades, falta de legislación, política y planes), recursos limitados o inadecuados (materiales educativos, no contar con suficientes profesores), problemas que tienen que ver con la escuela, como el currículo y la pedagogía, o la formación inadecuada de profesores (WHO, 2011, pp. 213-215).

Entre los factores o barreras identificados en este estudio que coinciden con la situación mundial, cabe resaltar algunos que el reporte mundial de WHO considera barreras significativas y que se desestiman en otros reportes:

-El apoyo de o hacia los profesores; en particular, cuando los grupos son muy grandes, un docente sin apoyo no puede manejar en el día a día las necesidades educativas de niños con discapacidad. Dado que el profesor de apoyo (educación especial) tiene hasta 27 niños que atender en distintos grupos, es muy difícil que pueda brindar ayuda de manera rutinaria a los docentes que imparten cada materia. 
- La falta de recursos y materiales educativos adecuados para enseñar los temas de las materias de ciencias, ya que esto hace que los estudiantes con discapacidad estén en riesgo de exclusión. Este tema es en particular relevante en la educación científica cuando se abordan aspectos abstractos o de carácter microscópico.

-Las barreras actitudinales, por ejemplo, la baja expectativa sobre los alumnos con discapacidad, como el no creer en la capacidad de los estudiantes para aprender. Esto no se limita a las personas con algún papel en la escuela, sino, incluso, ocurre en miembros de la familia del alumno. De hecho, el tema de las actitudes, sentimientos y preocupaciones de los docentes se aborda en el contexto mexicano con mayor profundidad en el artículo de RomeroContreras, Garcia-Cedillo, Forlin y Lomelí-Hernández (2013).

Una de las reflexiones importantes que surge desde este análisis es la enorme complejidad de la educación inclusiva de las ciencias en el sistema actual. De ahí la relevancia y necesidad de que las políticas públicas y sus instrumentos sean planeados cuidadosa y detalladamente para asegurar que exista una correspondencia entre los objetivos y la ruta para lograrlos. Sin duda, se deben, además, considerar los principios del diseño universal para hacer posible una educación para todos.

Una manera de pensar en alternativas para atender a los alumnos con necesidades educativas especiales es a través de los lentes conceptuales de la teoría de la cognición distribuida (Cole, 1998; Giere \& Moffatt, 2003; Gutierrez \& Vossoughi, 2010). Desde esta perspectiva, se pueden idear opciones en las que los docentes no carguen con toda la responsabilidad de la educación inclusiva. Esto implica conformar una comunidad de práctica cuyos miembros piensen juntos acerca de la dimensión del reto que puede representar el atender con eficacia la diversidad para, así, diseñar soluciones en las que, por medio de la división del trabajo, se atiendan e integren a todos los participantes en la misma actividad educativa; por ejemplo, diferentes alumnos pueden tomar turnos de interacción con los alumnos con necesidad de ser incluidos; se pueden diseñar de antemano guías de interacción en actividades situadas; e incorporar a todos los profesores del consejo técnico en la planeación de estrategias de inclusión que involucren a toda la escuela y descarguen al docente de grupo o al de apoyo de la exclusividad de esta consigna.

El espíritu de la perspectiva de cognición distribuida es que el todo es más complejo que la suma de las contribuciones individuales, y desde esa postura se pueden repensar las acciones específicas que convengan de manera más estratégica a cada escuela. Al reconocer la naturaleza distribuida de la cognición, y aplicarla en el diseño curricular, los educadores demuestran que la identificación de un problema, más allá de su solución, es una competencia crucial para manejar la complejidad de los retos que enfrentan, incluyendo la colaboración con alumnos con necesidades especiales.

Desde la lógica de la cognición distribuida, a continuación presentamos un conjunto de contradicciones que se deben atender, y enfocarse en la manera en la que los artefactos, los roles y la división del trabajo construyen una comunidad de participantes interesados en potenciar las capacidades de alumnos con discapacidades físicas:

Reynaga-Peña / Fernández-Cárdenas. La educación científica de alumnos con discapacidad visual Sinéctica 53 www.sinectica.iteso. $m x$ 
-Invertir tiempo suficiente para la preparación docente con la finalidad de que los profesores se apropien de los principios pedagógicos más adecuados y los puedan aplicar para hacer realidad una escuela para todos, que atienda la diversidad de perfiles de alumnos que asisten.

- Preparar a los docentes en las posibilidades de creación y adaptación de materiales con una perspectiva de diseño universal para el aprendizaje, que favorezca el trabajo colaborativo entre alumnos con diferentes necesidades.

- Mejorar las formas de colaboración entre los profesores del aula regular y los profesores de educación especial mediante la complementariedad de funciones y la comunicación eficiente. Evitar competencias de funciones y deslinde de responsabilidades compartidas.

- Capacitar a los docentes en contenidos científicos y de didáctica de la ciencia que les permitan ir más allá de los temas presentados en los libros de texto. El libro de texto es un primer insumo, necesario, pero no suficiente para una educación STEM de calidad.

- Trabajar en forma colegiada el manejo reflexivo de prejuicios de los docentes sobre las supuestas limitaciones cognitivas de los alumnos con discapacidades físicas. No ser omisos, negligentes o sobreprotectores con ellos, dados estos prejuicios.

- Construir lecciones y actividades educativas con otros medios semióticos que posibiliten que los alumnos se sientan desafiados en sus capacidades cognitivas. Evitar privilegiar siempre el uso de textos escritos; otros medios semióticos son posibles, por ejemplo, braille, lenguaje de señas, macrotipos, etcétera.

- Involucrar a padres de familia de modo especializado para que puedan colaborar con los docentes del aula regular y con los docentes de educación especial. Aprovechar su interés en el desarrollo de sus hijos, y no marginarlos.

Estas acciones contribuirán a optimizar la forma en que los participantes coadyuven en implementar una educación inclusiva de calidad basada en la conformación de sistemas situados de actividad. En estos sistemas, artefactos y participantes construyen significados de manera intersubjetiva con los medios a su alcance. La reflexión conjunta y crítica de los aspectos señalados conducirá a una mejor calidad de la educación con una perspectiva de inclusión.

\section{Agradecimientos}

Agradecemos a las personas que voluntariamente nos compartieron sus experiencias y puntos de vista sobre el tema aquí tratado, ya sea por medio de las entrevistas o conversaciones informales. Este trabajo puede dar cuenta de los retos que enfrenta la educación inclusiva gracias a los directivos y docentes de las escuelas que nos abrieron las puertas para poder observar las clases. Gracias a Irais Monserrat Santillán Rosas por su apoyo para revisar este documento. 


\section{REFERENCIAS BIBLIOGRÁFICAS}

American Chemical Society (ACS). Committee on Chemists with Disabilities (2001).Teaching chemistry to students with disabilities: A manual for high schools, colleges, and graduate programs (4a. ed.). D. L. Miner, R. Nieman, A. B. Swanson \& M. Woods (eds).

Atwood, R. K. \& Oldham, B. R. (1985). Teachers' perceptions of mainstreaming in an inquiry oriented elementary science program. Science Education, vol. 69, núm. 5, pp. 619-624. doi: 10.1002/sce.3730690504

Cole, M. (1998). Cultural psychology: A once and future discipline. Cambridge, MA: Belknap Press of Harvard University Press.

Corbin, J. \& Strauss, A. (1990). Grounded theory research: Procedures, canons and evaluative criteria. Qualitative Sociology, pp. 3-21. doi: 10.1007/ BF00988593

Engeström, Y. (2005). The discursive construction of collaborative care. En Y. Engeström (ed.). Developmental work research: Expanding activity theory in practice (pp 399-436). Köln: Lehmanns Media.

Flores-Camacho, F. (coord.) (2012). La enseñanza de la ciencia en la educación básica en México. México: Instituto Nacional para la Evaluación de la Educación. Recuperado de http://publicaciones.inee.edu.mx/buscadorPub/ P1/C/227/P1C227.pdf

Giere, R. N. \& Moffatt, B. (2003). Distributed cognition: Where the cognitive and the social merge. Social Studies of Science, vol. 33, núm. 2, pp. 301-310. doi: $10.1177 / 03063127030332017$

Goodwin, C. (1997). The blackness of black: Color categories as situated practice. En L. B. Resnick, R. Säljö, C. Pontecorvo \& B. Burge (eds.). Discourse, tools, and reasoning: Essays on situated cognition (pp. 111-140). Berlin: SpringerVerlag.

Gutierrez, K. D. \& Vossoughi, S. (2010). Lifting off the ground to return anew: Mediated praxis, transformative learning, and social design experiments. Journal of Teacher Education, vol. 61, núm. 1-2, pp. 100-117. doi: $10.1177 / 0022487109347877$

Hutchins, E. (1995). Cognition in the wild. MIT Press.

Instituto Nacional para la Evaluación de la Educación (INEE) (2015). Panorama educativo de México. Indicadores del Sistema Educativo Nacional. Educación básica y media superior. México. Recuperado de http://publicaciones.inee. edu.mx/buscadorPub/P1/B/114/P1B114.pdf

Instituto Nacional de Estadística y Geografía (INEGI) (2010). Censo Nacional de Población y Vivienda 2010. México.

Linn, M. (1972). An experiential science curriculum for the visually impaired. Exceptional Children, vol. 39, pp. 37-43. doi: 10.1177/001440297203900105

López Suero, C. C. et al. (2017). Ciencias experimentales en el aula inclusiva. En P. Membiela, N. Casado, M. I. Cebreiros y M. Vidal (eds.). La práctica docente en la enseñanza de las ciencias (pp. 59-65). Educación Editora.

Malone, L. \& De Lucchi, L. (1979). Life science for visually impaired students. Science and Children, vol. 16, núm. 5, pp. 29-31.

Maxwell, J. A. (2013). Qualitative research design: An interactive approach. Thousand Oaks, California: Sage Publications Inc. 
McGinnis, J. R. (2013). Teaching science to learners with special needs. Theory into Practice, vol. 52, pp. 43-50. doi: 10.1080/07351690.2013.743776

Reynaga-Peña C. G. et al. (2018). Creating a dialogic environment for transformative science teaching practices: Towards an inclusive education for science. Journal of Education for Teaching, vol. 44, núm. 1, pp. 44-57. doi: 10.1080/02607476.2018.1422620

Reynaga-Peña, C. G. et al. (2014). Experiencias educativas en la enseñanza de las ciencias experimentales a niños y jóvenes con discapacidad visual. En Memorias del Congreso Iberoamericano de Ciencia, Tecnología, Innovación y Educación (artículo 938). Recuperado de https://www.oei.es/historico/congreso2014/memoriactei/938.pdf

Romero-Contreras, S. y García Cedillo, I. (2013). Educación especial en México. Desafíos de la educación inclusiva. Revista Latinoamericana de Educación Inclusiva, vol. 7, núm. 2, pp. 1-9. Recuperado de https://dialnet.unirioja.es/ servlet/articulo?codigo $=4752911$

Romero-Contreras, S., Garcia-Cedillo I., Forlin C. \& Lomelí-Hernández K. A. (2013). Preparing teachers for inclusion in Mexico: How effective is this process? Journal of Education for Teaching: International Research and Pedagogy, vol. 39, núm. 5, pp. 509-522. doi:10.1080/02607476.2013.836340

Secretaría de Educación Pública (2017). Modelo Educativo para la Educación Obligatoria. Ciudad de México. Recuperado de https://www.gob.mx/cms/ uploads/attachment/file/207252/Modelo_Educativo_OK.pdf

Secretaría de Educación Pública (2011). Artículo 592 por el que se establece la Articulación de la Educación Básica. México, DF. Recuperado de http://dof. gob.mx/nota_detalle.php?codigo $=5205518 \&$ fecha $=19 / 08 / 2011$

Soler-Martí, M. A. (1999). Didáctica multisensorial de las ciencias: un nuevo método para alumnos ciegos, deficientes visuales y también sin problemas de visión. Barcelona: Ed. Paidós.

Stefanich, G. (2001). On the outside looking in. En G. Stefanich (ed.). Teaching in inclusive classrooms, theory and foundations. Washington, DC: National Science Foundation.

United Nations (2006). Convention on the Rights of Persons with Disabilities. Nueva York. Recuperado de http://www.un.org/disabilities/convention/ conventionfull.shtml

United Nations Educational, Scientific and Cultural Organization (Unesco) (2009). Education for all global monitoring report. París.

United Nations Educational, Scientific and Cultural Organization (Unesco) (2000). The Dakar framework for action: Education for All: Meeting our collective commitments. Adopted by the World Education Forum, Dakar, United Nations Educational, Scientific and Cultural Organization. 26-28 de abril. París. Recuperado de https://unesdoc.unesco.org/ark:/48223/pf0000121147_spa

Wilson, M. (2002). Six views of embodied cognition. Psychonomic Bulletin and Review, vol. 9, núm. 4, pp. 625- 636. doi: 10.3758/BF03196322

World Health Organization (WHO) (2011). World Report on Disability. Geneva: Recuperado de http://whqlibdoc.who.int/publications/2011/9789240685215_eng.pdf 